\title{
Prospective associations between maternal stress during pregnancy and fasting glucose with obstetric and neonatal outcomes
}

\author{
Antje Horsch $^{\mathrm{a}, \mathrm{b}, *}$, Leah Gilbert ${ }^{\mathrm{b}}$, Stefano Lanzi ${ }^{\mathrm{c}, \mathrm{d}}$, Ji Seon Kang ${ }^{\mathrm{c}}$, Yvan Vial ${ }^{\mathrm{b}}$, Jardena J. Puder ${ }^{\mathrm{b}}$ \\ ${ }^{a}$ Institute of Higher Education in Healthcare Research, University of Lausanne, Lausanne, Switzerland \\ ${ }^{\mathrm{b}}$ Department Woman-Mother-Child, Lausanne University Hospital, Lausanne, Switzerland \\ ' Service of Endocrinology, Diabetes and Metabolism, Lausanne University Hospital, Lausanne, Switzerland \\ ${ }^{\mathrm{d}}$ Division of Angiology, Heart and Vessel Department, Lausanne University Hospital, Switzerland
}

\section{A R T I C L E I N F O}

\section{Keywords:}

Gestational diabetes mellitus

Stress

Glucose

Obstetric

Neonatal

Depression

\begin{abstract}
A B S T R A C T
Objective: This prospective study investigated associations between maternal stress exposure and maternal psychological stress measures during pregnancy with obstetric and neonatal outcomes. We also tested whether any observed associations would be moderated by increasing glucose levels, as increased glycaemia is also associated with adverse obstetric and neonatal outcomes.

Methods: 203 women between 24 and 30 weeks gestation completed validated questionnaires assessing pregnancy-related major events and major life events, maternal perceived stress, and depression, anxiety, and stress symptoms. Glucose was measured using fasting morning blood samples. Instrumental delivery represented an obstetric outcome. Neonatal outcomes included Apgar score, large and small for gestational age weight, cord blood pH, NICU hospitalization, and neonatal hypoglycaemia.

Results: Regarding the obstetric outcome, pregnancy-related major life events OR $=1.346$ (1.016-1.783; $p=.016)$ were related to more incidences of instrumental delivery. Regarding neonatal outcomes, exposure to major life events in the last 12 months was associated with lower cord blood pH values $\mathrm{B}=-0.155$ ( -0.059 to $-0.002 ; p=.036)$ and with more incidences of hypoglycaemia $\mathrm{OR}=0.165(0.012-0.169 ; p=.04)$. Maternal psychological stress measures were related to more incidences of instrumental delivery $\mathrm{OR}=1.018$ (1.003-1.032; $p=.013)$. Maternal stress perception was associated with higher cord blood $\mathrm{pH}$ values $\mathrm{B}=0.155$ $(0-0.003 ; p=.046)$ and fewer NICU hospitalisations OR $=-0.170(-0.009$ to $-0.001 ; p=.019)$. Some of these associations between life events and stress perceptions with neonatal outcomes were moderated by fasting glucose levels.

Conclusion: Maternal pregnancy events as well as stress, depression and anxiety symptoms have a negative impact on obstetric outcomes and maternal life events are associated with negative neonatal outcomes. Higher fasting glucose levels moderate some of the relationships between stress and neonatal outcomes.
\end{abstract}

\section{Introduction}

Pregnancy is a time when women are confronted with particular stressors linked to physical alterations; hormonal changes that are often associated with rapid changes in mood, and pregnancy-specific anxiety linked to anticipating the pain of giving birth or fearing for the wellbeing of their baby [1]. Any physical or psychosocial stimulus can be stressful, provided that it is perceived as threatening for the homeostasis and the survival of the person [2]. Maternal stress exposure and psychological stress measures (perceived stress, and symptoms of stress, anxiety, and depression) are some of the most commonly assessed components of maternal stress [3,4].

Prenatal maternal stress exposure and stress perception are associated with less favourable obstetric outcomes, such as caesarean section [5-7]. Few studies have also demonstrated that stressful life events are related to certain neonatal outcomes such as low birth weight $[8,9]$ and to delayed lactogenesis [10] but, to our knowledge, associations of life events with other neonatal outcomes have so far not been investigated.

Although the exact physiological mechanisms by which maternal stress exposure may affect their offspring are still under investigation, two mechanisms have been suggested. One pathway is the transmission

\footnotetext{
* Corresponding author at: Institute of Higher Education in Healthcare Research, University of Lausanne, SV-A, Route de la Corniche 10, Lausanne CH-1010, Switzerland.

E-mail address: antje.horsch@chuv.ch (A. Horsch).
} 
of maternal stress hormones, particularly glucocorticoids, across the placenta [11]. The other pathway is linked to reduced uterine blood flow [4].

Apart from maternal stress, increased maternal glucose levels, as observed in women with gestational diabetes mellitus (GDM) can also have adverse effects on obstetric (e.g., instrumental delivery) and neonatal (e.g., LGA, hypoglycaemia, NICU hospitalization) outcomes [12-14]. In a recent study, negative obstetric or neonatal outcomes occurred in $71 \%$ of women with GDM [15]. In another study, approximately $60 \%$ of women with GDM gave birth by caesarean section and between $5 \%$ and $20 \%$ of their infants were admitted to the NICU [13]. Adverse obstetric outcomes can be explained by the excess of glucose that passes the placenta and that stimulates insulin production in the foetus, leading to excessive weight gain in the foetus and a higher prevalence of LGA, thus rendering vaginal delivery more complicated [16-18]. Importantly, the relationship between glycaemia and most adverse outcomes is continuous [19]. These outcomes gain increasing importance in view of the increasing prevalence of GDM [20] and it is therefore pertinent to examine whether associations between maternal prenatal stress variables and neonatal outcomes are moderated by maternal glycaemic levels.

This study aimed to firstly investigate associations between maternal stress exposure (pregnancy-unrelated major life events, pregnancy-related major events) and maternal psychological stress measures (perceived stress and symptoms of anxiety, depression, and stress) during pregnancy with obstetric and neonatal outcomes. Secondly, we tested whether these associations would be moderated by fasting glucose levels, as increased glycaemia is also associated with adverse obstetric and neonatal outcomes. We hypothesised that higher maternal stress exposure and maternal stress responses were related to more adverse obstetric and neonatal outcomes and that observed associations would be moderated by fasting glucose levels.

\section{Methods}

\subsection{Participant consent and recruitment}

The study took place in the Department of Obstetrics and Gynaecology of a Swiss University Hospital. First data of this cohort were previously reported [21]. Pregnant women taking part in routine screening for GDM with a 75-g OGTT at 24 to 30 weeks of gestation were given an information sheet and had the opportunity to ask questions about the study. After obtaining signed consent, a blood sample was taken for routine analysis of fasting 1-and 2-h glucose levels. Finally, participants completed three self-report questionnaires, they were weighed in light clothes and without shoes, and their height was measured. Shortly after childbirth, information on obstetric and neonatal outcomes was taken from the hospital record.

The Cantonal Ethics Committee of Vaud provided ethical approval (study number 295/12). Women were excluded if they were not able to complete the self-report questionnaires due to French language difficulties or if they had medical problems (such as chronic infections, autoimmune disease, asthma, renal insufficiency) and/or used medications (such as prednisone) that could both influence either their cortisol and/or glucose levels.

\subsection{Measures: maternal stress variables}

\subsubsection{Maternal stress exposure}

Pregnancy-related major events and major life events. Participants were given a list of three negative pregnancy-related major events (suspected growth retardation, vaginal bleeding, premature contractions) as well as ten negative major life events (death of someone they were close to, serious illness, exposure to abuse, exposure to violence, serious accident, unemployment, disability, alcohol/drug abuse, divorce, moving house) and were asked whether they had been exposed to any of these events in the last 12 months [22,23].

\subsubsection{Psychological stress measures}

Depression, Anxiety and Stress Scale-21 (DASS-21) [24]. The severity of symptoms of depression, anxiety and stress in the last week were measured with the DASS-21 on a 4-point Likert scale $(0=$ did not apply to me at all; 3 = applied to me very much or most of the time). A total score was calculated by summing up the sub-scores [25]. Adequate psychometric properties were demonstrated [26]. In the present study, Cronbach's $\alpha$ of the total score was $\alpha=0.91$.

Perceived Stress Scale (PSS [27]).The level of cognitive stress perception in the last month was rated on a 5-point Likert scale $(0=$ never; $4=$ very often). A total PSS score was obtained by reversing seven positive items and then summing across all 14 items. Adequate psychometric properties were reported [27]. Cronbach's $\alpha$ of the PSS in the present study was $\alpha=0.77$.

\subsubsection{Obstetric outcomes}

From the hospital record, information was collected regarding parity and instrumental delivery (including forceps, vacuum extraction, caesarean section; yes/no).

\subsubsection{Neonatal outcomes}

Information regarding the gestational age, Apgar scores at $5 \mathrm{~min}$, birth weight, LGA (weight) and SGA (weight) at birth [28], cord blood $\mathrm{pH}$ (venous), whether the newborn was hospitalized in the NICU, whether the newborn had hypoglycaemia ( $\leq 2.5 \mathrm{mmol} / \mathrm{L}$ ), and whether the newborn was breastfed (no vs mixed or exclusive) at hospital discharge were taken from the hospital record. The 5 min Apgar score was chosen as a proxy for the assessment of the infant's risk [29].

\subsubsection{GDM diagnosis}

GDM diagnosis was assessed using a 75-g oral glucose-tolerance test at 24-30 weeks of gestation and diagnosed if one of the following criteria applied according to the International Association of the Diabetes and Pregnancy Study Groups (IADPSG): fasting glucose $\geq 5.1 \mathrm{mmol} / \mathrm{L}$, l-h glucose $\geq 10.0 \mathrm{mmol} / \mathrm{L}$, or 2 -h glucose $\geq 8.5 \mathrm{mmol} / \mathrm{L}$ [30-32]. To assess the moderation effect in this study, we used the fasting glucose values at this time point. As the link between glycaemia and adverse outcomes is continuous, we chose to use rather the continuous variable (fasting glucose levels) than the categorical variable (GDM diagnosis) to assess moderation. In addition, cut-offs differ between different national and international recommendations are controversial and have changed over the last years.

2.2.6. Maternal sociodemographic, medical, and anthropometric variables

Age, education, pre-pregnancy weight and height were assessed by questionnaires. Current weight and height were assessed by physical exam. Current medication was extracted from the hospital record.

\subsection{Data analysis}

All analyses were carried out with SPSS Version 23.0 (SPSS, Inc., Chicago, IL, USA). For all variables, normality of distribution was tested. Linear or logistic regression analyses were computed, with obstetric and neonatal outcomes as dependent variables and maternal stress exposure and maternal psychological stress measures as independent variables. For the logistic regression analyses, odds ratios were used; for the linear regression analyses, the standardised Betas were reported. Adjustments for the following confounding variables were made: for the obstetric outcome (instrumental delivery): Model 1; maternal age and GDM diagnosis, second step, Model 2: Model 1 and additionally gestational age, birth weight, and maternal BMI during pregnancy. For neonatal outcomes, all analyses were done with the following adjustments: Model 1: gestational age, baby's sex, maternal age and GDM diagnosis; second step, Model 2: Model 1 and additionally 
Table 1

Maternal sociodemographic, obstetric, and neonatal variables.

\begin{tabular}{|c|c|c|}
\hline & $M(S D)$ & $n(\%)$ \\
\hline \multicolumn{3}{|c|}{ Maternal sociodemographic and anthropometric variables } \\
\hline Age (years) & $30.28(5.6)$ & \\
\hline Parity & $0.67(0.9)$ & \\
\hline \multicolumn{3}{|l|}{ Educational background } \\
\hline Compulsory education not completed & & $8(3.9)$ \\
\hline Compulsory education completed & & $54(26.6)$ \\
\hline Apprenticeship & & $49(24.1)$ \\
\hline Secondary school & & $16(7.9)$ \\
\hline University degree & & $76(37.4)$ \\
\hline BMI before pregnancy $\left(\mathrm{kg} / \mathrm{m}^{2}\right)$ & $23.26(4.8)$ & \\
\hline BMI at $24-30$ wks GA $\left(\mathrm{kg} / \mathrm{m}^{2}\right)$ & $26.57(4.8)$ & \\
\hline GDM diagnosis & & $39(19.2)$ \\
\hline \multicolumn{3}{|l|}{ Obstetric variables } \\
\hline Instrumental delivery & & $76(39.4)$ \\
\hline \multicolumn{3}{|l|}{ Neonatal variables } \\
\hline Gestational age (weeks) & $39.96(2.1)$ & \\
\hline APGAR score $5 \mathrm{~min}$ & $9.35(1.1)$ & \\
\hline Birth weight (in grams) & $3250.24(504.5)$ & \\
\hline LGA (weight) & & $19(9.9)$ \\
\hline SGA (weight) & & $24(12.5)$ \\
\hline Cord Blood Ph (Venous) & $7.34(0.1)$ & \\
\hline NICU Hospitalization & & $12(6.3)$ \\
\hline Hypoglycaemia & & $8(4.2)$ \\
\hline
\end{tabular}

BMI: body mass index; LGA: large for gestational age (yes/no); SGA: small for gestational age (yes/no); NICU hospitalization: yes/no; Hypoglycaemia: $\leq 2.5 \mathrm{mmol} / \mathrm{L}$.

maternal BMI during pregnancy. Finally, we tested whether these associations in the respective Models 1 were moderated by fasting glucose levels using linear and logistic regression models.

\section{Results}

\subsection{Sample characteristics}

Three-hundred and twenty-six consecutive women attended routine screening. Of these, 77 were excluded due to language difficulties. The study was presented to the remaining 249 patients and 25 declined to participate. In a second step, of the remaining 224 women, 21 were excluded due to medical problems, such as diseases necessitating cortisone intake and thus interfering with the analyses. Therefore, 203 women participated in the study. Thirty-nine $(19.2 \%)$ of these participants were diagnosed with GDM. Women had a mean age of 30.28 (5.6) years. Thirty-seven percent had a university degree, $27 \%$ had completed their compulsory education, and $24 \%$ their apprenticeship. Nearly $40 \%$ of participants had an instrumental delivery (see Table 1 for sociodemographic data). Data on maternal BMI during pregnancy was missing for 9, gestational age for 10, and major life events for 19 participants. Missing data was not replaced.

\subsection{Associations between maternal stress exposure and obstetric or neonatal outcomes}

Obstetric outcomes: There was a positive relationship between pregnancy-related major events $\mathrm{OR}=1.346(1.016-1.783 ; p=.016)$ and incidences of instrumental delivery. This association no longer remained significant after further adjustments (gestational age, birth weight, and maternal BMI; Table 2).

Neonatal outcomes: Exposure to major life events in the past 12 months and was related to lower venous blood $\mathrm{pH}$ values $\mathrm{B}=-0.155(-0.059$ to $-0.002 ; p=.036)$. This remained significant after further adjustments $\mathrm{B}=-0.173(-0.062$ to $-0.005 ; p=.023)$. Exposure to major life events in the past 12 months was associated with more incidences of neonatal hypoglycaemia OR $=0.165(0.012-0.169$; $p=.04)$ that also remained significant after further adjustments $\mathrm{OR}=0.176(0.015-0.165 ; p=.019)$. There were no significant relationships between maternal stress exposure variables and other neonatal outcomes (all $p=\mathrm{ns}$; Table 2).

\subsection{Associations between maternal psychological stress measures and obstetric or neonatal outcomes}

Obstetric outcomes: Regarding psychological stress measures, there was a positive relationship between DASS total $\mathrm{OR}=1.018$ $(1.003-1.032 ; p=.013)$ and incidences of instrumental delivery. This association no longer remained significant after further adjustments (gestational age, birth weight, and maternal BMI; Table 3).

Neonatal outcomes: There was a positive association between maternal cognitive stress perception and higher cord blood $\mathrm{pH}$ (venous) $\mathrm{B}=0.155(0-0.003 ; p=.046)$ which was no longer significant after further adjustment (maternal BMI). A significant relationship was found for maternal cognitive stress perception and fewer NICU hospitalizations $\mathrm{OR}=-0.170(-0.009$ to $-0.001 ; p=.019)$. This remained significant after adjustment $\mathrm{OR}=-0.158(-0.009-0 ; p=.015)$. There were no significant relationships between other psychological stress measures and other neonatal outcomes (all $p=$ ns; Table 3).

3.4. Influence of fasting glucose as a moderator on the relationships between maternal stress and obstetric or neonatal outcomes

Obstetric outcomes: There was no significant moderation effect of fasting glucose on the relationship between maternal stress exposure or psychological stress measures with instrumental delivery (all $p=\mathrm{ns}$; Table 4).

Neonatal outcomes: In the presence of higher fasting glucose levels, pregnancy-related major events were associated with a lower risk of NICU hospitalization OR $=0.261(0.084-0.813 ; p=.02)$ and major life events in the past twelve months were associated with lower frequency of the offspring being born LGA (weight) $\mathrm{OR}=0.004$ (0-0.640; $p=.033$ ). In the presence of higher fasting glucose values, maternal cognitive stress perception during pregnancy was associated with a lower APGAR scores $\mathrm{B}=-0.151(-0.031-0 ; p=.047)$, a higher frequency of the offspring being born LGA (weight) $B=1.262$ (1.013-1.573; $p=.038$ ) and a lower frequency of SGA (weight) $\mathrm{OR}=0.872(0.76-1 ; p=0.049)$. Finally, in the presence of increasing fasting glucose, maternal cognitive stress perception was related to lower frequency of NICU hospitalizations $\mathrm{OR}=0.832(0.708-0.978$; $p=0.026$; Table 4).

\section{Discussion}

This study investigated associations between maternal stress exposure (major life events, pregnancy-related major events), maternal psychological stress measures (cognitive stress perception and symptoms of anxiety, depression, and stress) during pregnancy and obstetric and neonatal outcomes in a cohort of women presenting for routine oral glucose tolerance testing at a University Hospital. Regarding obstetric outcomes, significant positive associations between pregnancy-related major events and incidences of instrumental delivery were found. Regarding neonatal outcomes, exposure to major life events was associated with lower cord blood $\mathrm{pH}$ values and more frequent neonatal hypoglycaemia. Maternal psychological stress, anxiety and depression (DASS) symptoms were associated with higher incidences of instrumental delivery. Maternal cognitive stress perception was associated with more favourable neonatal outcomes, i.e., higher cord blood $\mathrm{pH}$ values and fewer NICU hospitalizations. Several associations between life or pregnancy events and/or cognitive stress perception, but not DASS, with neonatal outcomes were moderated by fasting glucose levels. However, in contrast to our initial assumptions, those moderating effects were partly protective with regards to neonatal outcomes. 
Table 2

Stepwise linear or logistic regression analyses with birth and neonatal outcomes as dependent variables and maternal stress exposure as independent variable.

\begin{tabular}{|c|c|c|c|c|}
\hline & \multicolumn{2}{|l|}{ Model 1} & \multicolumn{2}{|l|}{ Model 2} \\
\hline & Pregnancy-related major events & Major life events in the past 12 months & Pregnancy-related major events & Major life events in the past 12 months \\
\hline \multicolumn{5}{|l|}{ Obstetric outcomes } \\
\hline Instrumental delivery ${ }^{\mathrm{a}}$ & $1.346(1.016-1.783)_{*}$ & $1.228(0.519-2.902)$ & $1.208(0.887-1.645)$ & $1.403(0.554-3.557)$ \\
\hline \multicolumn{5}{|l|}{ Neonatal outcomes } \\
\hline APGAR $5 \mathrm{~min}$ & $0.044(-0.108-0.196)$ & $-0.125(-0.83-0.058)$ & $0.043(-0.113-0.199)$ & $-0.139(-0.881-0.028)$ \\
\hline LGA (weight) ${ }^{\mathrm{a}}$ & $-0.006(-0.044-0.04)$ & $-0.018(-0.139-0.107)$ & $-0.006(-0.045-0.041)$ & $-0.007(-0.132-0.119)$ \\
\hline SGA (weight) ${ }^{\mathrm{a}}$ & $-0.019(-0.053-0.041)$ & $0.122(-0.019-0.255)$ & $-0.006(-0.049-0.045)$ & $0.127(-0.018-0.254)$ \\
\hline Cord Blood Ph (Venous) & $0.077(-0.005-0.015)$ & $-0.155(-0.059--0.002)_{*}$ & $0.076(-0.005-0.015)$ & $\begin{array}{l}-0.173(-0.062- \\
-0.005)_{*}\end{array}$ \\
\hline NICU Hospitalization ${ }^{\mathrm{a}}$ & $-0.074(-0.046-0.014)$ & $0.136(-0.002-0.170)$ & $-0.084(-0.048-0.012)$ & $0.119(-0.014-0.162)$ \\
\hline Hypoglycaemia $^{\text {a }}$ & $-0.038(-0.036-0.021)$ & $0.165(0.012-0.169)_{*}$ & $-0.015(-0.030-0.025)_{\text {水* }}$ & $0.176(0.015-0.165)_{*}$ \\
\hline
\end{tabular}

LGA: large for gestational age (yes/no); SGA: small for gestational age (yes/no); NICU hospitalization: yes/no; Hypoglycaemia: $\leq 2.5 \mathrm{mmol} / \mathrm{L}$.

Adjustments in obstetric outcomes: Model 1: maternal age and GDM diagnosis; Model 2: maternal age, GDM diagnosis, gestational age, birth weight (in grams) and mother's BMI during pregnancy.

Adjustments in neonatal outcomes: Model 1: gestational age, baby's sex, maternal age and GDM diagnosis; Model 2: gestational age, baby's sex, maternal age, GDM diagnosis and mother's BMI during pregnancy.

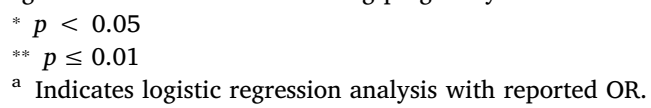

Table 3

Stepwise linear or logistic regression analyses with birth and neonatal outcomes as dependent variables and maternal psychological stress responses as independent variable.

\begin{tabular}{|c|c|c|c|c|}
\hline & \multicolumn{2}{|l|}{ Model 1} & \multicolumn{2}{|l|}{ Model 2} \\
\hline & DASS total & Maternal stress perception & DASS total & Maternal stress perception \\
\hline \multicolumn{5}{|l|}{ Obstetric outcomes } \\
\hline Instrumental delivery ${ }^{\mathrm{a}}$ & $1.018(1.003-1.032)_{*}$ & $1.021(0.981-1.063)$ & $1.009(0.993-1.025)$ & $0.997(0.952-1.044)$ \\
\hline \multicolumn{5}{|l|}{ Neonatal outcomes } \\
\hline APGAR $5 \mathrm{~min}$ & $0.024(-0.006-0.009)$ & $0.055(-0.013-0.029)$ & $0.031(-0.006-0.009)$ & $0.062(-0.014-0.031)$ \\
\hline LGA (weight) ${ }^{\mathrm{a}}$ & $0.001(-0.002-0.002)$ & $0.032(-0.005-0.007)$ & $-0.037(-0.003-0.002)$ & $-0.011(-0.007-0.006)$ \\
\hline SGA (weight) ${ }^{\mathrm{a}}$ & $0.041(-0.002-0.003)$ & $0.003(-0.006-0.007)$ & $0.081(-0.001-0.004)$ & $0.046(-0.005-0.009)$ \\
\hline Cord Blood Ph (Venous) & $0.102(0-0.001)$ & $0.155(0-0.003)_{*}$ & $0.09(0-0.001)$ & $0.141(0-0.003)$ \\
\hline NICU Hospitalization ${ }^{\mathrm{a}}$ & $-0.104(-0.003-0)$ & $-0.170(-0.009 \text { to }-0.001)_{*}$ & $-0.087(-0.003-0.001)$ & $-0.158(-0.009-0)_{*}$ \\
\hline Hypoglycaemia $^{a}$ & $-0.085(-0.002-0.001)$ & $-0.063(-0.006-0.002)$ & $-0.043(-0.002-0.001)_{* *}$ & $-0.032(-0.005-0.003)$ \\
\hline
\end{tabular}

LGA: large for gestational age (yes/no); SGA: small for gestational age (yes/no); NICU hospitalization: yes/no; Hypoglycaemia: $\leq 2.5 \mathrm{mmol} / \mathrm{L}$.

Adjustments in obstetric outcomes: Model 1: maternal age and GDM diagnosis; Model 2: maternal age, GDM diagnosis, gestational age, birth weight (in grams) and mother's BMI during pregnancy.

Adjustments in neonatal outcomes: Model 1: gestational age, baby's sex, maternal age and GDM diagnosis; Model 2: gestational age, baby's sex, maternal age, GDM diagnosis and mother's BMI during pregnancy.

${ }^{*} p<0.05$

*** $p \leq 0.01$

${ }^{\mathrm{a}}$ Indicates logistic regression analysis with reported OR.

Table 4

Stepwise linear or logistic regression analyses with birth and neonatal outcomes as dependent variables and maternal stress.

\begin{tabular}{|c|c|c|c|c|}
\hline & Pregnancy-related major events & Major life events in the past 12 months & DASS total & Maternal stress perception \\
\hline \multicolumn{5}{|l|}{ Birth outcomes } \\
\hline Instrumental delivery ${ }^{\mathrm{a}}$ & $0.829(0.462-1.488)$ & $98.655(0.81-12,016.091)$ & $0.993(0.967-1.019)$ & $0.978(0.896-1.068)$ \\
\hline \multicolumn{5}{|l|}{ Neonatal outcomes } \\
\hline APGAR $5 \mathrm{~min}$ & $0.314(-0.238-0.369)$ & $-1.358(-1.990-0.137)$ & $0.072(-0.004-0.008)$ & $-0.151(-0.031-0)_{\text {米 }}$ \\
\hline LGA (weight) ${ }^{\mathrm{a}}$ & $1.389(0.477-4.045)$ & $0.004(0-0.640)_{*}$ & $1.046(0.983-1.113)$ & $1.262(1.013-1.573)_{*}$ \\
\hline SGA (weight) ${ }^{\mathrm{a}}$ & $1.114(0.507-2.45)$ & $15.27(0.842-277.026)$ & $0.958(0.911-1.008)$ & $0.872(0.76-1)_{*}$ \\
\hline Cord Blood Ph (Venous) & $-0.095(-0.013-0.001)$ & $-0.344(-0.083-0.053)$ & $0.117(0-0.001)$ & $0.017(-0.001-0.001)$ \\
\hline NICU Hospitalization ${ }^{\mathrm{a}}$ & $0.261(0.084-0.813)_{*}$ & $0.732(0.029-18.213)$ & $0.976(0.935-1.019)$ & $0.832(0.708-0.978)_{*}$ \\
\hline Hypoglycaemia $^{\mathrm{a}}$ & $1.412(0.452-4.414)$ & $0.299(0.007-12.589)$ & $0.956(0.851-1.074)_{\text {水* }}$ & $0.957(0.784-1.169)$ \\
\hline
\end{tabular}

LGA: large for gestational age (yes/no); SGA: small for gestational age (yes/no); NICU hospitalization: yes/no; Hypoglycaemia: $\leq 2.5$ mmol/L.

Adjustments in obstetric outcomes: maternal age and GDM diagnosis

Exposure and maternal psychological stress responses as independent variables, with fasting glucose as moderator

Adjustments in neonatal outcomes: gestational age, baby's sex, maternal age and GDM diagnosis.

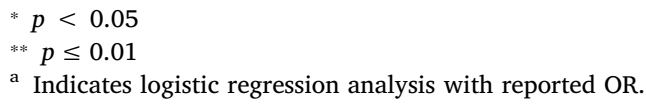


Some of our results fit in with previous findings reporting associations between pregnancy life events, instrumental delivery, and other delivery complications [33]. A novel finding is the association between major life events and lower cord blood $\mathrm{pH}$ values that to our knowledge has not been previously investigated. This could possibly be explained by the fact that the more a mother is exposed to stress during pregnancy, the more the foetus is in presence of his mother's stress hormones, via cortisol passing through the placenta, which in turn can lower the cord blood $\mathrm{pH}$ values [34].

The association between major life events and neonatal hypoglycaemia is also, to our knowledge, a novel finding. This could also be explained by an increase in maternal stress and stress regulatory hormonal responses throughout pregnancy, e.g., in glucagon, catecholamines, and cortisol, which can increase maternal glucose levels at any time during the day. Glucose crosses the placenta, stimulating fetal insulin production, thus increasing the risk of hypoglycemia as the umbilical cord is cut [35].

The positive associations between psychological stress, anxiety and depression measures (DASS total) and incidences of instrumental delivery that we found are in line with previous research. For example, two studies identified that prenatal anxiety and depression were associated with instrumental delivery [36,37].

Our results also showed a positive, i.e., protective correlation between maternal cognitive stress perception and higher cord blood $\mathrm{pH}$, although this disappeared when controlling for maternal BMI. This correlation is surprising because, as explained before, maternal emotional stress can heighten foetal stress, which then lowers cord blood $\mathrm{pH}$ $[34,38]$. Similarly, the association we found between maternal cognitive stress perception and reduced NICU hospitalizations is in contrast with a review reporting a relationship between prenatal stress and suboptimal infant outcome [1]. However, NICU hospitalization had not been assessed specifically [1]. Evidence showing that as pregnancy progresses, women perceive major events as less stressful compared with earlier on in pregnancy might help to explain these results [39]. For example, maternal cognitive stress perception during early pregnancy but not later on was associated with shorter gestational length [39].

Given that increased maternal glucose levels are known to be associated with less favourable obstetric and neonatal outcomes, we tested whether the associations between maternal prenatal stress variables and neonatal outcomes are moderated by maternal glycaemic levels to see if a combination of potentially adverse predictors would augment the risk of less favourable outcomes. We found that certain relationships were amplified/augmented, whilst certain were reduced when introducing fasting glucose as a moderator. To our knowledge, the role of fasting glucose as a moderator of the relationship between stress exposure or perception with neonatal outcomes has not been previously tested. We observed moderation effects when life events, but mainly when maternal cognitive stress perception was the predictor. When adding fasting plasma glucose as a moderator, we found a reduction in the association between pregnancy-related major events and NICU hospitalization. Thus, neonates of women with more stressful pregnancy events were less susceptible to being hospitalized after birth the more elevated their mother's fasting glucose levels was. Furthermore, neonates of women with higher previous stress exposure to life events were less likely to be born with a higher birth weight (LGA) the more elevated their mother's fasting glucose levels are. This points surprisingly to a potentially protective impact of glucose levels in a certain range on the effect of stress exposure to certain neonatal outcomes, although higher glucose levels per se are related to a higher frequency of LGA children [14].

Regarding the role of glucose as a moderator of the relationship of maternal cognitive stress perception and neonatal outcomes, we had both protective as well as amplifying effects: We found that neonates of women with higher cognitive stress perception during pregnancy had lower APGAR scores ( $5 \mathrm{~min}$ ) the more elevated their maternal fasting glucose levels were. Fasting plasma glucose levels per se have been previously found to be unrelated to APGAR score at $1 \mathrm{~min}$ [40], but its effect on moderating the impact of maternal cognitive stress perception on APGAR has not been tested so far. We also showed that neonates of women with higher cognitive stress perception during pregnancy are more likely to be born with LGA (weight) and less likely with SGA (weight) the more elevated their mother's glucose levels are. As maternal hyperglycemia increases the risk of LGA in the offspring [14] and also decreases the risk of SGA [41], this effect is not surprising and might be beneficial in the setting of stress, as maternal stress increases the risk of having a SGA baby $[38,42]$. Increased maternal glycaemia in our observed ranges was also protective on the impact of maternal cognitive stress perception on NICU hospitalization. Potentially, women with higher glucose values know about their higher risk and are perhaps more careful about taking care of themselves, especially if they perceive this risk. The above results should be investigated in future studies and help to identify women whose infants are most at risk of adverse outcomes.

The findings of our study should be interpreted with caution because of some limitations. Major life and pregnancy-related events were reported retrospectively, and thus, may be biased. Psychological stress measures were obtained by self-report only, although these are validated, standardised questionnaires. Furthermore, it would have been helpful to include the participants' BMI at delivery into the analyses. However, this is not a routine clinical measurement and this data therefore cannot be found in the women's hospital record. A major strength of our study was the assessment before the blood draws and before participants found out about their GDM diagnosis, thus avoiding a potential bias of emotions linked to the diagnosis on stress measures and also avoiding a recall bias towards life events. To increase external validation, this study included both women with and without GDM. However, as women with GDM got more specific care and advice, we adjusted all our analyses for the diagnosis of GDM.

Our results demonstrate the critical role of maternal stress on obstetric and neonatal outcomes [4]. Thus, this study has important clinical implications, suggesting that screening pregnant women for stress exposure, stress emotional symptoms, and cognitive stress perception early on in pregnancy and offering support to those in need may contribute to preventing some negative obstetric and neonatal outcomes. However, the role of maternal stress exposure and especially cognitive stress perception seems to be more complex than previously thought and might also depend on whether other factors such as the women's glucose levels are taken into consideration or not. In some instances, higher glycaemia levels within a certain range might even have a protective impact on the stress-outcome relationships that need to be further investigated in future studies.

\section{Declaration of Competing Interest}

The authors have no competing interests to report

\section{Acknowledgements}

We would like to thank all participants and Dr. Andrea Meyer for his assistance regarding the interpretation of the mediation analyses. This study was funded by a grant from the Swiss Diabetes Foundation and represents a pilot study for the Swiss National Science Foundation project \#32003B_176119.

\section{References}

[1] E.J. Mulder, P.G. Robles de Medina, A.C. Huizink, B.R. Van den Bergh, J.K. Buitelaar, G.H. Visser, Prenatal maternal stress: effects on pregnancy and the (unborn) child, Early Hum. Dev. 70 (1-2) (2002) 3-14.

[2] R.S. Lazarus, From psychological stress to the emotions: a history of changing outlooks, Annu. Rev. Psychol. 44 (1993) 1-21.

[3] A.C. Huizink, P.G. de Medina, E.J. Mulder, G.H. Visser, J.K. Buitelaar, Psychological 
measures of prenatal stress as predictors of infant temperament, J. Am. Acad. Child Adolesc. Psychiatry 41 (9) (2002) 1078-1085.

[4] B.R. Van den Bergh, E.J. Mulder, M. Mennes, V. Glover, Antenatal maternal anxiety and stress and the neurobehavioural development of the fetus and child: links and possible mechanisms. A review, Neurosci. Biobehav. Rev. 29 (2) (2005) 237-258.

[5] J. Martini, S. Knappe, K. Beesdo-Baum, R. Lieb, H.-U. Wittchen, Anxiety disorders before birth and self-perceived distress during pregnancy: associations with maternal depression and obstetric, neonatal and early childhood outcomes, Early Hum. Dev. 86 (5) (2010) 305-310.

[6] T.A. Saunders, M. Lobel, C. Veloso, B.A. Meyer, Prenatal maternal stress is associated with delivery analgesia and unplanned cesareans, J. Psychosom. Obstet. Gynecol. 27 (3) (2006) 141-146.

[7] P. Zhu, J. Hao, X. Jiang, K. Huang, F. Tao, New insight into onset of lactation: mediating the negative effect of multiple perinatal biopsychosocial stress on breastfeeding duration, Breastfeed. Med. 8 (2) (2013) 151-158.

[8] D.D. Costa, M. Dritsa, J. Larouche, W. Brender, Psychosocial predictors of labor/ delivery complications and infant birth weight: a prospective multivariate study, J. Psychosom. Obstet. Gynecol. 21 (3) (2000) 137-148.

[9] C. Taberna, Foetal and neonatal stress, J. Matern. Fetal Neonatal Med. 25 (sup4) (2012) 136-137.

[10] K.G. Dewey, Maternal and fetal stress are associated with impaired lactogenesis in humans, J. Nutr. 131 (11) (2001) 3012s-3015s.

[11] P. Ghaemmaghami, S.M. Dainese, R. La Marca, R. Zimmermann, U. Ehlert, The association between the acute psychobiological stress response in second trimester pregnant women, amniotic fluid glucocorticoids, and neonatal birth outcome, Dev. Psychobiol. 56 (4) (2014) 734-747.

[12] S. Easmin, T. Chowdhury, M. Islam, A. Beg, M. Jahan, T. Latif, et al., Obstetric outcome in early and late onset gestational diabetes mellitus, Mymensingh Med. J. 24 (3) (2015) 450-456.

[13] S. Ghosh, K. Ghosh, Maternal and neonatal outcomes in gestational diabetes mellitus, J. Indian Med. Assoc. 111 (5) (2013) 330-1, 6.

[14] Z. Wang, L. Kanguru, J. Hussein, A. Fitzmaurice, K. Ritchie, Incidence of adverse outcomes associated with gestational diabetes mellitus in low-and middle-income countries, Int. J. Gynecol. Obstet. 121 (1) (2013) 14-19.

[15] J.S. Park, D.W. Kim, J.-Y. Kwon, Y.W. Park, Y.H. Kim, H.Y. Cho, Development of a screening tool for predicting adverse outcomes of gestational diabetes mellitus: a retrospective cohort study, Medicine. 95 (1) (2016).

[16] A. Blachier, C. Alberti, D. Korb, T. Schmitz, V. Patrick, B. Christine, et al., Diet or medically treated gestational diabetes: is there any difference for obstetrical and neonatal complications? A French cohort study, J. Perinat. Med. 42 (3) (2014) 315-319.

[17] M. Boulvain, O. Irion, T. Dowswell, J.G. Thornton, Induction of labour at or near term for suspected fetal macrosomia, Cochrane Libr 5 (2016).

[18] G. Vilchez, J. Dai, L. Hoyos, N. Gill, R. Bahado-Singh, R. Sokol, Labor and neonatal outcomes after term induction of labor in gestational diabetes, J. Perinatol. 35 (11) (2015) 924.

[19] B.E. Metzger, L.P. Lowe, A.R. Dyer, E.R. Trimble, U. Chaovarindr, D.R. Coustan, et al., Hyperglycemia and adverse pregnancy outcomes, N. Engl. J. Med. 358 (19) (2008) 1991-2002.

[20] A. Horsch, J. Gross, F.R. Jornayvaz, S. Lanzi, J.J. Puder, Gestational diabetes-what are the non-medical approaches? Rev Med. Suisse 12 (521) (2016) 1089-1091.

[21] A. Horsch, J.S. Kang, Y. Vial, U. Ehlert, A. Borghini, P. Marques-Vidal, et al., Stress exposure and psychological stress responses are related to glucose concentrations during pregnancy, Br. J. Health Psychol. 21 (3) (2016) 712-729.

[22] F.S. Koch, A. Sepa, J. Ludvigsson, Psychological stress and obesity, J. Pediatr. 153 (6) (2008) 839-844.
[23] C. Obel, M. Hedegaard, T.B. Henriksen, N.J. Secher, J. Olsen, S. Levine, Stress and salivary cortisol during pregnancy, Psychoneuroendocrinology. 30 (7) (2005) 647-656.

[24] P.F. Lovibond, S.H. Lovibond, The structure of negative emotional states: comparison of the Depression Anxiety Stress Scales (DASS) with the Beck Depression and Anxiety Inventories, Behav. Res. Ther. 33 (3) (1995) 335-343.

[25] A. Osman, J.L. Wong, C.L. Bagge, S. Freedenthal, P.M. Gutierrez, G. Lozano, The depression anxiety stress scales-21 (DASS-21): further examination of dimensions, scale reliability, and correlates, J. Clin. Psychol. 68 (12) (2012) 1322-1338.

[26] J.D. Henry, J.R. Crawford, The short-form version of the Depression Anxiety Stress Scales (DASS-21): construct validity and normative data in a large non-clinical sample, Br. J. Clin. Psychol./Br. Psychol. Soc. 44 (Pt 2) (2005) 227-239.

[27] S. Cohen, T. Kamarck, R. Mermelstein, A global measure of perceived stress, J. Health Soc. Behav. 24 (4) (1983) 385-396.

[28] J. Villar, L.C. Ismail, C.G. Victora, E.O. Ohuma, E. Bertino, D.G. Altman, et al., International standards for newborn weight, length, and head circumference by gestational age and sex: the Newborn Cross-Sectional Study of the INTERGROWTH21st Project, Lancet 384 (9946) (2014) 857-868.

[29] K. Thorngren-Jerneck, A. Herbst, Low 5-minute apgar score: a population-based register study of 1 million term births, Obstet. Gynecol. 98 (1) (2001) 65-70.

[30] I. Blumer, E. Hadar, D.R. Hadden, L. Jovanovic, J.H. Mestman, M.H. Murad, et al., Diabetes and pregnancy: an endocrine society clinical practice guideline, J. Clin. Endocrinol. Metab. 98 (11) (2013) 4227-4249.

[31] D.R. Coustan, L.P. Lowe, B.E. Metzger, A.R. Dyer, The Hyperglycemia and adverse pregnancy outcome (HAPO) study: paving the way for new diagnostic criteria for gestational diabetes mellitus, Am. J. Obstet. Gynecol. 202 (6) (2010) 654.e1-6.

[32] 13. Management of diabetes in pregnancy: standards of medical care in diabetes2018, Diabetes Care 41 (Suppl. 1) (2018) S137-s43.

[33] M. Lobel, Conceptualizations, measurement, and effects of prenatal maternal stress on birth outcomes, J. Behav. Med. 17 (3) (1994) 225-272.

[34] F.T. Lim, S.A. Scherjon, J.M. van Beckhoven, A. Brand, H.H. Kanhai, J.M. Hermans, et al., Association of stress during delivery with increased numbers of nucleated cells and hematopoietic progenitor cells in umbilical cord blood, Am. J. Obstet. Gynecol. 183 (5) (2000) 1144-1151.

[35] P.C. Maheux, B. Bonin, A. Dizazo, P. Guimond, D. Monier, J. Bourque, et al., Glucose homeostasis during spontaneous labor in normal human pregnancy, J. Clin. Endocrinol. Metab. 81 (1) (1996) 209-215.

[36] J. Alder, N. Fink, J. Bitzer, I. Hösli, W. Holzgreve, Depression and anxiety during pregnancy: a risk factor for obstetric, fetal and neonatal outcome? A critical review of the literature, J. Matern. Fetal Neonatal Med. 20 (3) (2007) 189-209.

[37] T.K. Chung, T.K. Lau, A.S. Yip, H.F. Chiu, D.T. Lee, Antepartum depressive symptomatology is associated with adverse obstetric and neonatal outcomes, Psychosom. Med. 63 (5) (2001) 830-834.

[38] R.M. Reynolds, Corticosteroid-mediated programming and the pathogenesis of obesity and diabetes, J. Steroid Biochem. Mol. Biol. 122 (1) (2010) 3-9.

[39] L.M. Glynn, P.D. Wadhwa, C. Dunkel-Schetter, A. Chicz-Demet, C.A. Sandman, When stress happens matters: effects of earthquake timing on stress responsivity in pregnancy, Am. J. Obstet. Gynecol. 184 (4) (2001) 637-642.

[40] G. Sesmilo, E. Meler, V. Perea, I. Rodríguez, A. Rodríguez-Melcón, M. Guerrero, et al., Maternal fasting glycemia and adverse pregnancy outcomes in a Mediterranean population, Acta Diabetol. 54 (3) (2017) 293-299.

[41] T.J. Rosenberg, S. Garbers, H. Lipkind, M.A. Chiasson, Maternal obesity and diabetes as risk factors for adverse pregnancy outcomes: differences among 4 racial/ ethnic groups, Am. J. Public Health 95 (9) (2005) 1545-1551.

[42] C. Hobel, J. Culhane, Role of psychosocial and nutritional stress on poor pregnancy outcome, J. Nutr. 133 (5) (2003) 1709S-1710S. 